\title{
Saúde e meio ambiente: análise de diferenciais intra-urbanos, Município de São Paulo, Brasil*
}

\author{
Environment and health: an analysis of intra-urban differentials in the \\ City of S. Paulo, Brazil
}

\author{
Marco Akerman, Paulo Campanario e Paulo Borlina Maia \\ Centro de Estudos de Cultura Contemporânea. São Paulo - SP, Brasil (M. A.), Fundação SEADE - \\ Diretoria Adjunta de Estudos Populacionais. São Paulo, SP - Brasil (P.C., P.B.M.)
}

\begin{abstract}
Resumo
Objetivou-se preencher as lacunas no tipo de informação necessária sobre mortalidade para estabelecimento de correlações entre saúde, urbanização e meio ambiente. As estatísticas produzidas baseiam-se na análise de dados de registros contínuos, dados de pesquisas, relatórios e censos populacionais. Dados socioeconômicos foram utilizados para construir um indicador composto para cada distrito e subdistrito fornecendo a base para a construção de quatro zonas socioeconômicas. São apresentados diferenciais de mortalidade entre essas zonas, distribuídos por quatro grupos de causas e cinco grandes grupos etários. O zoneamento urbano mostrou que $43,8 \%$ da população da cidade de São Paulo vive em áreas com as piores condições socioeconômicas. Os dados de saúde demonstraram que pessoas vivendo nessas áreas tinham padrão de mortalidade mais elevado que aquelas vivendo em áreas com melhores condições socioeconômicas. Analisa-se a sobremortalidade entre as zonas socioeconômicas e sugere-se este enfoque como instrumento útil para a definição de prioridades na alocação de recursos de saúde.
\end{abstract}

Saúde urbana. Distribuição espacial. Mortalidade diferencial.

\begin{abstract}
A field study undertaken in the city of S. Paulo is presented as part of the project Environment and Health in Developing Countries: An Analisys of Intra-Urban Differentials Using Existing Data financed by the Ministry of Foreigh Affairs of the United Kingdom with academic support from the London School of Hygiene and Tropical Medicine (LSHTM). The research aim was to fill in the gaps in the information needed to establish associations between mortality, urbanization and the environment. Statistics were produced by means of existing data collected by city departments, research carried out by universities and census data. Data quality was assessed taking into consideration data coverage, accuracy, and sensibility to pinpoint deprived areas in the city of S. Paulo. Socioeconomic data were used to create a composite index for districts and subdistricst in order to form four socioeconomic zones. Mortality differentials between the zones by five broad age groups (0-4, 5-14, 15-44, 45-64 e 65+) and
\end{abstract}

\footnotetext{
* Trabalho apresentado no IV Congresso de Saúde Coletiva (ABRASCO) Recife, PE, 1994.

Correspondência para/Correspondence to: Marco Akerman - Centro de Estudos de Cultura Contemporânea/CEDEC - Rua Airosa Galvão, 64 05002-070 São Paulo, SP - Brasil. Fax: (011) 871.2123 E-mail: cedec@eu.amsp.br

Edição subvencionada pela FAPESP. Processo 95/2290-6.

Recebido em 21.6.95. Reapresentado em 30.3.1996. Aprovado em 15.4.1996.
} 
four ICD chapters (circulatory, respiratory, infectious and parasitic and external causes) are presented. The zoning used showed that $43.8 \%$ of S. Paulo residents live in areas under the worst socioeconomic conditions. Health data demonstrated that people living in this areas had higher rates of mortality then those living in the areas with the best conditions. Finally, excess mortality data are analysed and suggested as a good method for allocating health resources.

Urban health. Residence characteristics. Differential mortality.

\section{INTRODUÇÃO}

Pesquisadores paulistas já desenvolveram importantes trabalhos para medir diferenciais entre áreas geográficas urbanas $\left(\right.$ Leser $^{15}, 1972 ;$ Comune $^{6}$, 1982; FUNDAP $^{10}, 1983$; Ferreira e Grillo ${ }^{7}, 1983$; Bussab e Dini $^{3}$, 1985; Sobral ${ }^{22}$, 1988; Fundação SEADE ${ }^{11,12}$, 1988, 1990; Ramos e Goihman ${ }^{19}$, 1989; Secretaria de Planejamento e Gestão ${ }^{20}$, 1993), mas há ainda algumas lacunas a serem preenchidas.

Em primeiro lugar, os trabalhos até agora concentraram-se mais em diferenciais regionais do que nos intra-urbanos. Provavelmente o espaço urbano é mais sensível para mostrar diferenças sociais do que o espaço regional*. Além disso, não houve tentativa sistematizada para descrever diferenciais por sexo e por todos os grupos etários em relação ao grupo social. Por exemplo, não há estudo publicado comparando padrões de mortalidade com condições socioeconômicas para a população adulta (19 a 65), desagregados para todas as regiões geográficas dentro do Município de São Paulo. E, finalmente, mesmo quando mortalidade e diferenciais socioeconômicos foram descritos, há importantes lacunas quanto a sexo, idade e desagregação geográfica.

Em 1991, o Banco Mundial, através do seu Urban Management Program, encomendou à London School of Hygiene nad Tropical Medicine uma revisão crítica de artigos que abordassem impactos do meio ambiente e das condições socioeconômicas sobre a saúde nos países em desenvolvimento. Essa revisão (Bradley e col. ${ }^{2}, 1992$ ), que resultou na seleção de 100 artigos referentes ao assunto, apontou a predominância da investigação desses impactos em relação às crianças e em detrimento da análise das conseqüências sobre a saúde da população adulta; que as doenças diarreicas são as mais pesquisadas, e que há poucos estudos com relação às doenças respiratórias. Constatou-se ainda que grande parte dos artigos abordam o meio urbano como um todo, baseando as suas análises em grandes agregados estatísticos, sem dar muita atenção aos diferenciais intraurbanos**.

O presente estudo busca preencher as lacunas no tipo de informação necessária sobre mortalidade para o estabelecimento de correlações entre saúde, urbanização e meio ambiente. $\mathrm{O}$ presente artigo originase da pesquisa de campo realizada em São Paulo pelo projeto Environment and Health in Developing Countries: An Analysis of Intra-Urban Diferentials Using Existing Data*** (Stephens e col. ${ }^{23}$, 1994). O mencionado projeto desenvolveu-se em duas cidades com diferentes estágios de desenvolvimento econômico: Acra (Gana), 1992, e São Paulo, entre abril de 1993 e abril de 1994. A Diretoria Adjunta de Estudos Populacionais da Fundação SEADE foi a parceira na cidade de São Paulo.

Os objetivos específicos do estudo foram: (1) demonstrar a possibilidade de apontar diferenciais urbanos com o uso de dados existentes, a fim de realçar a reprodutividade da metodologia de trabalho para planejadores urbanos em outros países em desenvolvimento****; (2) obter a máxima desagregação geográfica dos dados de mortalidade por causas específicas, sexo, idade e áreas geográficas e correlacionálos com variáveis socioeconômicas e de ambiente; (3) produzir um instrumento simples e transparente para o gerenciamento urbano, que possibilite ilumi-

\footnotetext{
* Entende-se aqui o espaço regional como uma subdivisão estadual que abarca mais de um município, enquanto o espaço urbano é aqui considerado como espaço interno a um dado município.

** Dados socioeconômicos e de saúde são apresentados de forma agregada, descrevendo áreas muito abrangentes, como por exemplo o rural em oposição ao urbano. Este seria um dos motivos que explica serem as zonas urbanas consideradas, tradicionalmente, superiores às rurais, em termos de saúde e padrão de vida. Com efeito, análises muito agregadas limitam-se à situação média das pessoas e não detectam a enorme heterogeneidade existente dentro do próprio meio urbano (áreas de favelas ou cortiços versus bairros de classe média alta, etc). É também uma das razões que poderiam explicar por que os pobres residentes em áreas urbanas sejam olvidados nas políticas nacionais dirigidas à pobreza e às disparidades em saúde.

*** Financiada pelo Departamento de Meio Ambiente e Políticas do Overseas Development Administration (ODA), órgão do Ministério das Relações Exteriores do Reino Unido com o suporte acadêmico e institucional da Unidade de Saúde Urbana da London School of Hygiene and Tropical Medicine.

**** Já é lugar comum dizer que dados coletados rotineiramente possuem qualidade precária. Acreditamos que se formos capazes de demonstrar a utilidade desses dados talvez possamos criar bases motivacionais para o seu aperfeiçoamento.
} 
nar áreas prioritárias para intervenção e alocação de recursos; (4) instrumentalizar com dados empíricos a advocacia dos grupos postergados, viabilizando o que a literatura internacional chama de "discriminação positiva" de grupos humanos.

\section{METODOLOGIA}

As estatísticas produzidas basearam-se na análise de dados de registros contínuos, dados de pesquisas realizadas por universidades e órgãos de governo, relatórios publicados e não publicados, e de censos realizados por órgãos governamentais locais e nacionais. É de boa qualidade os dados existentes em São Paulo, particularmente para dados de mortalidade. Os dados socioambientais têm qualidade satisfatória e são capazes de apontar áreas de carência no espaço urbano. Entretanto, foram observadas algumas imperfeições nos dados estatísticos de registro contínuo em realçar carência ambiental. Alguns indicadores sociais e de ambiente como água, saneamento, e densidade habitacional não teriam sensibilidade suficiente, por exemplo, para apontar a existência de cortiços nas áreas centrais da cidade.

Os dados de mortalidade utilizados em São Paulo foram fornecidos pela Fundação SEADE, abarcando todos os óbitos ocorridos no município de São Paulo em 1992 62.355 óbitos. Os dados populacionais (população total e por faixa etária) foram obtidos através de estimativas feitas pela Fundação SEADE para o ano de 1992, com base no censo do IBGE de 1991, e na Pesquisa de Condições de Vida - PCV (Fundação SEADE ${ }^{13}$, 1992). Os dados socioeconômicos e de ambiente foram obtidos na Secretaria Municipal de Planejamento, na SABESP e do censo do IBGE de 1991.

A qualidade de todos esses dados foi analisada em função da cobertura (total, por área, por grupos específicos, por sexo e por idade) da acurácia (idade, causa, área) e da sua sensibilidade em apontar áreas de carência*.

A seleção das variáveis teve a participação de planejadores de 12 instituições públicas, municipais e estaduais, a partir da denominada "técnica de Delfos". O processo de escolha das variáveis socioeconômicas e de ambiente, e a discussão sobre a base geográfica adotada foram descritos em artigo publicado anteriormente pela Revista de Saúde Pública (Akerman e col. $\left.{ }^{1}, 1994\right)$. Foram selecionados cinco indicadores: renda familiar per capita; percentual de pessoas analfabetas e com primário incompleto; percentual de residências ligadas à rede de esgoto; consumo per capita de água e número de pessoas por domicílio. Esse número foi coletado, posteriormente, para cada um dos 56 distritos e subdistrito do município. Cada um desses indicadores foi transformado em valores que variaram de 0 a 1 , onde 0 indicava maior carência e 1 menor carência, semelhantes ao Índice de Desenvolvimento $\mathrm{Hu}$ mano (IDH) (United Nations ${ }^{24}$, 1992). Em seguida, foi feita uma média desses valores, o que gerou um ranking - do menos ao mais carente - de "índices compostos"** para todos os distritos e subdistritos. Finalmente, a cidade foi dividida em zonas homogêneas, segundo características socioeconômicas e de ambiente, por meio de quartis. $\mathrm{O}$ corte entre os quartis foi arbitrário, adotando-se a forma simplificada de número igual de distritos e subdistritos entre elas.

\section{RESULTADOS E COMENTÁRIOS}

\section{Diferenciais Socioeconômicos e de Ambiente}

A Tabela 1 apresenta as quatro zonas homogêneas e os respectivos índices compostos para cada distrito e subdistrito da cidade de São Paulo.

O subdistrito da Consolação apresentou o menor nível de carência, enquanto Perus se mostrou o subdistrito mais carente da cidade de São Paulo. Chama a atenção o fato de que $43,5 \%$ da população da cidade vive em áreas com as piores condições socioeconômicas e de ambiente (Zona 1), enquanto 9,2\% dos habitantes residem nas áreas com as melhores condições (Zona 4). Com esses percentuais, não se pretende inferir a quantidade de "pobres" ou "ricos" na cidade. Não se desconhece o fato de que há "bolsões de pobreza" na Zona 4 e "bolsões de riqueza" na Zona 1. Com esse tipo de desenho ecológico aqui utilizado, está-se apenas inferindo o risco coletivo de uma determinada área geográfica.

A Figura 1 mostra o mapa da distribuição dos distritos e subdistritos entre as quatro zonas homogêneas.

A Zona 4, é a que se apresenta com as melhores condições socioeconômicas e de ambiente, com seus subdistritos contigüamente localizados na área central da cidade. Ela vai sendo circundada gradualmente pelas outras três zonas, sendo que a Zona 1, com as piores condições socioeconômicas e de ambiente localiza-se na periferia da cidade. Esta distribuição das zonas replica a teoria dos anéis central, intermediário e periférico, apresentada por Leser $^{15}$ (1972).

A Tabela 2 apresenta os diferenciais socioeconômicos e de ambiente entre as quatro zonas homogêneas, confirmando os diferenciais entre as quatro

\footnotetext{
Maiores detalhes sobre essa análise podem ser obtidos junto aos autores do presente trabalho.

** Um Índice composto de carência é uma medida que associa diferentes variáveis num indicador sintético para analisar as características de grupos populacionais vivendo em determinadas áreas geográficas e que reflete as circunstâncias materiais ou sociais da área em questão (Carstairs e Morris, 19894; Locker, $1992^{16}$ ). A construção de um indicador composto através da combinação de variáveis ligadas a setores distintos dentro de uma administração urbana pode favorecer o diálogo intersetorial.
} 
Tabela 1 - Distribuição dos distritos e subdistritos da cidade de São Paulo entre as quatro zonas homogêneas.

\begin{tabular}{|c|c|c|}
\hline Zonas & Distritos & Índice Composto \\
\hline \multirow[t]{14}{*}{ Zona 4 - áreas com a melhores condições socioeconômicas e de ambiente } & Consolação & 0,849 \\
\hline & Cerqueira Cesar & 0,769 \\
\hline & Jardim Paulista & 0,720 \\
\hline & Jardim América & 0,717 \\
\hline & Indianópolis & 0,663 \\
\hline & Vila Mariana & 0,649 \\
\hline & Sé & 0,637 \\
\hline & Bela Vista & 0,634 \\
\hline & Pinheiros & 0,611 \\
\hline & Mooca & 0,606 \\
\hline & Santa Cecília & 0,596 \\
\hline & Perdizes & 0,591 \\
\hline & Barra Funda & 0,589 \\
\hline & Aclimação & 0,589 \\
\hline \multicolumn{2}{|l|}{ Percentagem de população vivendo na Zona 4} & $9,2 \%$ \\
\hline \multirow[t]{14}{*}{ Zone 3 - Primeira "áreas intermediárias" } & Vila Madalena & 0,566 \\
\hline & Belenzinho & 0,564 \\
\hline & Santa Ifigênia & 0,547 \\
\hline & Pari & 0,542 \\
\hline & Liberdade & 0,528 \\
\hline & Lapa & 0,516 \\
\hline & Ibirapuera & 0,493 \\
\hline & Cambuci & 0,481 \\
\hline & Alto da Mooca & 0,459 \\
\hline & Saúde & 0,459 \\
\hline & Bom Retiro & 0,458 \\
\hline & Brás & 0,437 \\
\hline & Casa Verde & 0,429 \\
\hline & Ipiranga & 0,419 \\
\hline \multicolumn{2}{|l|}{ Percentagem de população vivendo na Zona 3} & $13,8 \%$ \\
\hline \multirow[t]{14}{*}{ Zona 2 - Segunda "areas intermediárias" } & Tatuapé & 0,396 \\
\hline & Santana & 0,387 \\
\hline & Penha & 0,382 \\
\hline & Butantã & 0,379 \\
\hline & Jaguara & 0,376 \\
\hline & Vila Maria & 0,374 \\
\hline & N. Sra. do Ó & 0,365 \\
\hline & Limão & 0,363 \\
\hline & Vila Prudente & 0,356 \\
\hline & Vila Formosa & 0,343 \\
\hline & Vila Guilherme & 0,338 \\
\hline & Tucuruvi & 0,338 \\
\hline & Jabaquara & 0,331 \\
\hline & Pirituba & 0,315 \\
\hline \multicolumn{2}{|l|}{ Percentagem de população vivendo na Zona 2} & $33,2 \%$ \\
\hline \multirow[t]{14}{*}{ Zona 1 - Áreas com as piores condições socioeconômicas e de ambiente } & Vila Nova Cachoeir. & 0,314 \\
\hline & Cangaíba & 0,309 \\
\hline & Vila Matilde & 0,306 \\
\hline & Itaquera & 0,284 \\
\hline & Ermelino Matarazzo & 0,259 \\
\hline & Brasilândia & 0,254 \\
\hline & Santo Amaro & 0,235 \\
\hline & São Miguel Paulista & 0,228 \\
\hline & Guaianazes & 0,203 \\
\hline & Capela do Socorro & 0,178 \\
\hline & Itaim Paulista & 0,157 \\
\hline & Jaragüá & 0,148 \\
\hline & Parelheiros & 0,110 \\
\hline & Perus & 0,039 \\
\hline Percentagem de população vivendo na Zona 1 & & $43,8 \%$ \\
\hline
\end{tabular}




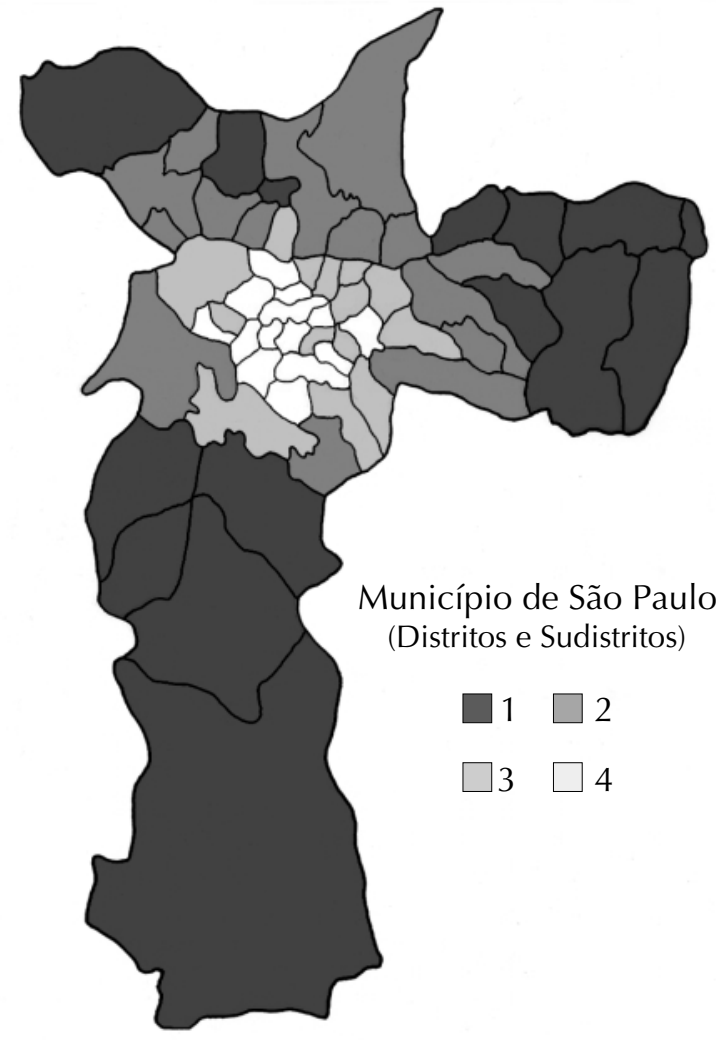

Figura 1 - Mapa dos distritos e subdistritos do Município de São Paulo, segundo zonas homogêneas (Confira Tabela1).

zonas da cidade de São Paulo. Os indicadores "renda familiar per capita" e consumo per capita de água são os que apresentam os maiores diferenciais entre as Zonas 4 e 1, respectivamente, 3,4 e 4,7 vezes.

\section{Diferenciais de Mortalidade entre as Zonas Homogêneas}

Os próximos tópicos ocupam-se da descrição dos diferenciais de quatro grupos selecionados de causas de morte:

- Doenças do aparelho circulatório;

- Doenças do aparelho respiratório;

- Doenças infecciosas e parasitárias;

- Causas externas.

Esses grupos foram desagregados pelas quatro zonas homogêneas e por cinco grandes grupos etários (0-4, 5-14, 15-44, 45-64 e 65 e +). O ano de referência foi 1992. No final do trabalho analisou-se a sobremortalidade entre as zonas homogêneas, sugerindo este enfoque como um instrumento útil para a definição de prioridades na alocação de recursos para a saúde.

\section{Mortalidade por Grupos de Causas no Grupo Etário 0-4 Anos}

A Tabela 3 mostra os diferenciais nas taxas de mortalidade por causa específica (/10.000), no grupo etário 0-4 anos, entre as zonas socioeconômicas e de ambiente.

Dos quatro grupos de causas escolhidos, as doenças respiratórias foram a principal causa de morte, cujos diferenciais são cinco vezes maiores se se compara a Zona 1 com a 4 (as taxas variam de 3,9, na Zona 4, a 14,9 na Zona 1, sendo esta última a de condições mais precárias). Leve-se em conta que os diferenciais entre essas duas zonas são estatisticamente significativos. Em segundo lugar, vêm as mortes do grupo de doenças infecciosas e parasitárias: os níveis

Tabela 2. - Diferenciais socioeconômicos e de ambiente entre as quatro zonas homogêneas.

\begin{tabular}{|c|c|c|c|c|c|}
\hline Zonas* & $\begin{array}{l}\text { Renda familiar per } \\
\text { capita média } \\
\text { (salários mínimos) }\end{array}$ & $\begin{array}{c}\text { Percentual médio } \\
\text { de pessoas } \\
\text { analfabetas e com } \\
\text { primário incompleto }\end{array}$ & $\begin{array}{l}\text { Percentual médio } \\
\text { de residências } \\
\text { ligadas à rede } \\
\text { rede de esgoto }\end{array}$ & $\begin{array}{l}\text { Consumo médio } \\
\text { mensal per capita } \\
\text { de água }\left(\mathrm{m}^{3}\right)\end{array}$ & $\begin{array}{c}\text { Número médio } \\
\text { de pessoas por } \\
\text { domicílio }\end{array}$ \\
\hline Zona 4 & 7,2 & 28,1 & 98,7 & 20,6 & 2,8 \\
\hline Zona 3 & 4,3 & 32,8 & 94,2 & 11,5 & 3,4 \\
\hline Zona 2 & 3,2 & 35,5 & 81,8 & 6,2 & 3,7 \\
\hline Zona 1 & 2,1 & 40,0 & 64,3 & 4,3 & 3,8 \\
\hline
\end{tabular}

Fontes: Fundação IBGE, 1991; Secretaria de Planejamento do Município de São Paulo, 1992; SABESP, 1993

* Confira Tabela 1

Tabela 3 - Taxas de mortalidade por causa específica (/10.000) por zonas para a faixa etária 0-4 anos, Município de São Paulo, 1992.

\begin{tabular}{lccccccccc}
\hline & População & \multicolumn{2}{c}{ Circulatório } & \multicolumn{2}{c}{ Respiratório } & \multicolumn{2}{c}{ Infecciosas } \\
Zonas* & 1992 & Óbitos & Taxas & Óbitos & Taxas & Óbitos & Taxas & Óbitos & Taxas \\
\hline 1 & 463.125 & 37 & 0,80 & 693 & 14,96 & 405 & 8,74 & 110 \\
2 & 310.246 & 29 & 0,93 & 258 & 8,32 & 110 & 3,55 & 51 \\
3 & 116.492 & 8 & 0,69 & 102 & 8,76 & 56 & 4,81 & 1,64 \\
4 & 87.059 & 5 & 0,57 & 34 & 3,91 & 14 & 1,61 & 1,03 \\
\hline Total & 976.922 & 79 & 0,81 & 1.087 & 11,13 & 585 & 5,99 & 191 \\
\hline
\end{tabular}

Fontes: Fundação SEADE ${ }^{13}$ (1992), STEPHENS, e col. ${ }^{23}$ (1994).

* Confira Tabela 1 
das taxas são aproximadamente a metade dos encontrados no grupo de doenças respiratórias, mas os diferenciais de taxas são igualmente importantes, acima de cinco vezes entre a Zona 1 e a 4 . Os diferenciais nas taxas de mortalidade por causas externas nas idades de 0-4 anos, não mostram diferenças nítidas entre zonas, e os níveis de mortalidade são muito baixos, em torno de 2 por 10.000 crianças. O grupo de doenças do aparelho circulatório também não apresentou diferenças nítidas entre as zonas e as taxas são ainda mais baixas. Nessas idades, tal tipo de causa de morte depende mais de defeitos congênitos do que do grupo social ou da zona socioeconômica da criança.

Os diferenciais de mortalidade em crianças com menos de cinco anos de idade são corroborados e complementados por evidências de outras pesquisas, como por exemplo, Monteiro ${ }^{17}$ (1982), Monteiro e Benício ${ }^{18}$ (1987), Ferreira e $\operatorname{Saad}^{8}$ (1988) e Ferreira9 ${ }^{9}$ (1989).

\section{Mortalidade por Causa Específica: Grupo Etário de 5 a 14 Anos}

A Tabela 4 mostra diferenciais de mortalidade no grupo etário de 5 a 14 anos.

De maneira geral, as taxas encontradas nessas idades foram muito baixas, sendo que as causas externas se destacaram por serem as mais altas. No entanto, o nível médio no Município de São Paulo, para esta causa, igual a 1,68 por 10.000 habitantes, ainda é baixo se comparado com o grupo que segue, de 15 a 44 anos, como se verá no próximo item.
Grosso modo, neste grupo de crianças e adolescentes, devido justamente ao pequeno número de casos, não se pode afirmar nada de categórico em termos de diferenças entre as zonas.

\section{Mortalidade por Causa Específica: Grupo Etário de 15 a 44 Anos}

A Tabela 5 e a Figura 2 mostram diferenciais de mortalidade em adultos em idade produtiva na cidade de São Paulo, em 1992.

As causas externas (mortes violentas) foram responsáveis pelas taxas de mortalidade mais altas (/10.000) para adultos em idade produtiva: no município como um todo, a taxa chegou a 12,9 por 10.000 habitantes, quase oito vezes mais elevada que a do

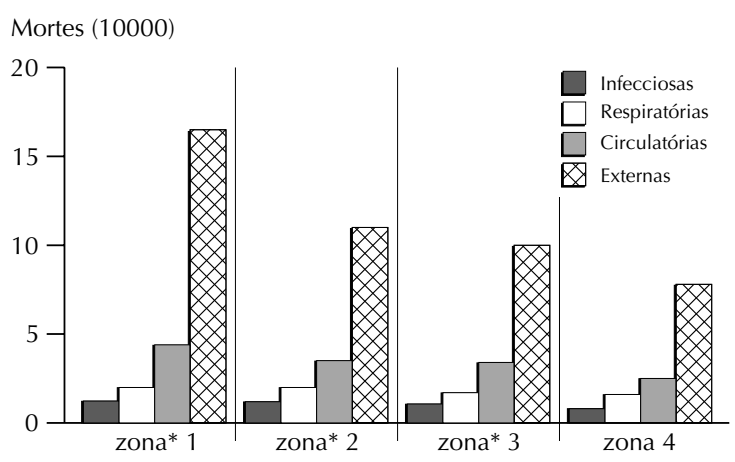

Figura 2 - Taxas de mortalidade por causa específica (/10.000) por zonas (conferir Tabela 1) para a faixa etária 15-44anos, Município de São Paulo.

Tabela 4 - Taxas de mortalidade por causa específica (/10.000) por zonas para a faixa etária 5-14 anos, Município de São Paulo, 1992.

\begin{tabular}{lccccccccc}
\hline & População & \multicolumn{2}{c}{ Circulatório } & \multicolumn{2}{c}{ Respiratório } & \multicolumn{2}{c}{ Infecciosas } & \multicolumn{2}{c}{ Externas } \\
Zonas* & 1992 & Óbitos & Taxas & Óbitos & Taxas & Óbitos & Taxas & Óbitos & Taxas \\
\hline 1 & 987.604 & 10 & 0,10 & 40 & 0,41 & 24 & 0,24 & 198 & 2,00 \\
2 & 674.532 & 10 & 0,15 & 24 & 0,36 & 14 & 0,21 & 93 & 1,38 \\
3 & 214.942 & 4 & 0,19 & 10 & 0,47 & 3 & 0,14 & 28 & 1,30 \\
4 & 102.193 & 1 & 0,10 & 8 & 0,78 & 2 & 0,20 & 13 & 1,27 \\
\hline Total & 1.979 .271 & 25 & 0,13 & 82 & 0,41 & 43 & 0,22 & 332 & 1,68 \\
\hline
\end{tabular}

Fontes: Fundação SEADE ${ }^{13}$ (1992), STEPHENS, e col. ${ }^{23}$ (1994).

* Confira Tabela 1

Tabela 5 - Taxas de mortalidade por causa específica (/10.000) por zonas para a faixa etária 15-44 anos, Município de São Paulo, 1992.

\begin{tabular}{lccccccccc}
\hline & População & \multicolumn{2}{c}{ Circulatório } & \multicolumn{2}{c}{ Respiratório } & \multicolumn{2}{c}{ Infecciosas } \\
Zonas* & 1992 & Óbitos & Taxas & Óbitos & Taxas & Óbitos & Taxas & Óbitos & Taxas \\
\hline 1 & 2.100 .893 & 922 & 4,39 & 414 & 1,97 & 256 & 1,22 & 3.428 \\
2 & 1.563 .283 & 546 & 3,49 & 303 & 1,94 & 184 & 1,18 & 1.696 \\
3 & 621.684 & 212 & 3,41 & 104 & 1,67 & 66 & 1,06 & 615 & 9,85 \\
4 & 420.169 & 104 & 2,48 & 65 & 1,55 & 36 & 0,86 & 326 \\
\hline Total & 4.706 .029 & 1.784 & 3,79 & 886 & 1,88 & 542 & 1,15 & 6.065 \\
\hline
\end{tabular}


grupo etário mais jovem, examinado no item anterior. A Zona 1 apresentou taxas de mortalidade para essa causa 2,1 vezes maior do que as taxas na Zona $4 *$. Em geral, as taxas de mortalidade por doenças do sistema circulatório são mais baixas que aquelas de causas externas. Elas apresentaram um gradiente similar e consistente entre as zonas. Residentes da Zona 1 apresentaram taxas de mortalidade por doenças circulatórias 1,7 superiores do que aqueles residentes na Zona 4. Vale observar que esse padrão de diferenciais (residentes em áreas socioeconômicas mais carentes tendo taxas de mortalidade mais altas do que os moradores das áreas mais privilegiados) é consistente com o padrão encontrado para adultos, em Acra (ver Stephens e col. ${ }^{23}$, 1994). Taxas de mortalidade para idades entre 15 e 44, anos por doenças infecciosas e respiratórias, também demonstraram diferenciais entre zonas; apesar de não serem muito acentuados.

\section{Mortalidade por Causa Específica: Grupo Etário de 45 a 64 Anos}

A Tabela 6 mostra os diferenciais de mortalidade nas idades entre 45 a 64 anos na cidade de São Paulo.

As doenças circulatórias apresentaram as taxas mais altas: 38,8 por 10.000 habitantes no município como um todo e diferenças significativas entre as zonas, que chegam a 1,7 vezes entre a 1 e a 4 . Os diferenciais entre zonas persistiram em todos os outros grupos de causas, apesar da menor importância relativa dos mesmos com respeito às doenças do aparelho circulatório**. A mortalidade por causas externas é relativamente mais baixa no grupo 45-64 anos, comparada com a de adultos jovens, mas é digno de nota que seus níveis sejam ainda muito elevados, de 7,75 por 10.000 no município, e os diferenciais ainda persistam e mostrem uma situação desfavorável para os residentes das zonas mais pobres.

\section{Mortalidade por Causa Específica: Grupo Etário de Mais de 65 Anos}

A Tabela 7 mostra os diferenciais de mortalidade para o grupo de mais de 65 anos na cidade de São Paulo.

As doenças circulatórias foram, de longe, o principal grupo de causas de morte: a taxa encontrada para o município foi de 232,68 por 10.000 habitantes. Não há, neste grupo etário, ao contrário dos anteriores, diferenças significativas entre as zonas, ou seja, parece ser que, no grupo 65 e mais anos, no caso das doenças circulatórias, os fatores socioeconômicos e de ambiente já não têm um peso tão importante na mortalidade. $\mathrm{O}$ grupo que se segue, do-

Tabela 6 - Taxas de mortalidade por causa específica (/10.000) por zonas para a faixa etária 45-64 anos, Município de São Paulo, 1992.

\begin{tabular}{|c|c|c|c|c|c|c|c|c|c|}
\hline \multirow[b]{2}{*}{ Zonas* } & \multirow{2}{*}{$\begin{array}{c}\text { População } \\
1992 \\
\end{array}$} & \multicolumn{2}{|c|}{ Circulatório } & \multicolumn{2}{|c|}{ Respiratório } & \multicolumn{2}{|c|}{ Infecciosas } & \multicolumn{2}{|c|}{ Externas } \\
\hline & & Óbitos & Taxas & Óbitos & Taxas & Óbitos & Taxas & Óbitos & Taxas \\
\hline 1 & 546.633 & 2.495 & 45,64 & 541 & 9,90 & 280 & 5,12 & 504 & 9,22 \\
\hline 2 & 519.572 & 2.155 & 41,48 & 449 & 8,64 & 186 & 3,58 & 379 & 7,29 \\
\hline 3 & 233.900 & 795 & 33,99 & 197 & 8,42 & 74 & 3,16 & 164 & 7,01 \\
\hline 4 & 195.952 & 515 & 26,28 & 117 & 5,97 & 44 & 2,25 & 113 & 5,77 \\
\hline Total & 1.496 .057 & 5.960 & 38,84 & 1.304 & 8,72 & 584 & 3,90 & 1.160 & 7,75 \\
\hline
\end{tabular}

Fontes: Fundação SEADE ${ }^{13}$ (1992), STEPHENS, e col. ${ }^{23}$ (1994).

* Confira Tabela 1

Tabela 7 - Taxas de mortalidade por causa específica (/10.000) por zonas para a faixa etária 65+ anos, Município de São Paulo, 1992.

\begin{tabular}{lccccccccc}
\hline & População & \multicolumn{2}{c}{ Circulatório } & \multicolumn{2}{c}{ Respiratório } & \multicolumn{2}{c}{ Infecciosas } & \multicolumn{2}{c}{ Externas } \\
Zonas* & 1992 & Óbitos & Taxas & Óbitos & Taxas & Óbitos & Taxas & Óbitos & Taxas \\
\hline 1 & 149.546 & 3.489 & 233,31 & 1.061 & 70,95 & 145 & 9,70 & 213 & 14,24 \\
2 & 187.190 & 4.616 & 246,59 & 1.448 & 77,35 & 204 & 10,90 & 250 & 13,36 \\
3 & 120.415 & 2.509 & 208,36 & 827 & 68,68 & 87 & 7,23 & 109 & 9,05 \\
4 & 91.965 & 2.163 & 235,20 & 735 & 79,92 & 65 & 7,07 & 109 & 11,85 \\
\hline Total & 549.116 & 12.777 & 232,68 & 4.071 & 74,14 & 501 & 9,12 & 681 & 12,40 \\
\hline
\end{tabular}

Fontes: Fundação SEADE13 ${ }^{13}$ (1992), STEPHENS, e col. ${ }^{23}$ (1994).

* Confira Tabela 1

\footnotetext{
* Vale a pena mencionar que os diferenciais homem/mulher para causas externas são os responsáveis pela maioria dos diferenciais de mortalidade em adultos jovens. As taxas para homens são 8 vezes mais altas do que de mulheres nas zonas 3 e 4 , e 10 e 12 vezes nas zonas 1 e 2 respectivamente. A sobremortalidade masculina permanece quando avaliamos acidentes de tráfego e homicídios. Diferenciais homem/mulher são mais visíveis em taxas de homicídios (as taxas são 19 vezes maiores para homens do que para mulheres na zona 1). Não há diferenciais importantes entre as zonas para taxas de acidentes de trânsito em homens, mas as taxas de homicídio masculinas são 3 vezes mais altas na zona 1 do que na zona 4 .

** As doenças circulatórias apresentam taxas masculinas mais altas em todas as zonas.
} 
enças respiratórias, não mostrou, igualmente, nenhum padrão de diferencial entre zonas. Note-se que é unicamente neste grupo etário que os padrões de mortalidade não mostram diferenças substanciais e consistentes entre zonas. As taxas de mortalidade por doenças infecciosas consistituem o único grupo no qual os padrões seguem tendência com alguma consistência de diferenciais, das áreas mais privilegiadas às mais carentes.

\section{Taxas de Mortalidade Padronizadas por Idade por Causa Específica, segundo Zona}

Para complementar a análise por grupos etários, a Tabela 8 apresenta taxas padronizadas por idade, por causa específica segundo zonas socioeconômicas e de ambiente. Além disso, o risco de morte segundo causa para residentes nas Zonas 1, 2 e 3 são comparados aos riscos relativos de morte para residentes na Zona 4 (risco relativo ou RR).

A Tabela 8 evoca a transição epidemiológica do Município de São Paulo, similar à das áreas urbanas do Brasil. Pode-se observar diferenças sempre consistentes entre a mortalidade por causas e as zonas, ou seja, quanto mais desprivilegiada em termos socioeconômicos, maior a mortalidade, apesar de que as diferenças podem ser maiores ou menores segundo a causa. As causas de morte por doenças circulatórias apresentaram as taxas mais altas, representando cerca de um terço da Taxa Bruta de Mortalidade (indicada como "Todas", na Tabela 8) e, por outro lado, as diferenças entre zonas, medidas através dos riscos relativos, variam de 1,00 (Zona 4) a 1,21, na Zona 1, passando por 1,01 (Zona 3) e 1,19 (Zona 2). As causas externas (mortes violentas) mostraram diferenças muito grandes entre as zonas, com os índices variando de 1,00 (Zona 4) a 1,86, ou seja, quase o dobro (Zona 4). No caso das doenças respiratórias, os índices variaram de 1,00 a 1,20. As causas infecciosas foram as menos importantes, mas com os maiores diferenciais entre zonas, que variam de 1,00 a 2,10, ou seja, mais de duas vezes. Os riscos relativos de mortalidade entre zonas mostraram diferenciais maiores no caso de doenças infecciosas e causas externas. As diferenças na Taxa Bruta de Mortalidade variaram de 1,00 , na Zona 4 , a 1,18 , na Zona 1 .

\section{Sobremortalidade entre as Zonas}

A sobremortalidade entre as zonas pode ser observada nas Tabelas 9, 10 e 11. Seu cálculo é feito da seguinte maneira: a) escolheu-se, para cada idade e causa, a zona com a taxa de mortalidade mais baixa (ZonaPadrão, em geral a Zona 4); b) multiplicou-se a taxa da Zona-Padrão pela população de cada uma das outras três zonas, chegando-se, assim, ao número de mortos esperados de cada zona, ou seja, ao número de mortos que ocorreriam em cada zona se sua mortalidade fosse igual à da Zona-Padrão; c) calculou-se a diferença entre os mortos observados e os esperados, que pode ser definida como sobremortalidade absoluta; d) dividiuse a sobremortalidade absoluta pelo número de mortos observados, chegando-se à sobremortalidade relativa, ou seja, à percentagem de mortes evitáveis naquela zona, por determinada causa e grupo etário, se nela imperassem as mesmas condições socioeconômicas e de ambiente da Zona-Padrão.

Presume-se a partir da Tabela 9 que $55 \%$ das mortes que ocorreram na Zona 1 , no grupo etário de 0 a 4 anos, poderiam ser evitadas se a Zona 1 tivesse as mesmas condições socioeconômicas da Zona 4. Considerando a cidade como um todo, conclui-se que 2.442 crianças não teriam morrido se a cidade tivesse um perfil socioeconômico e de ambiente uniforme.

Tabela 8 - Taxas de mortalidade padronizadas e riscos relativos (RR) por causa específica (/10.000) por zonas, Município de São Paulo, 1992.

\begin{tabular}{ccccccccccc}
\hline & \multicolumn{2}{c}{ Circulatórias } & \multicolumn{2}{c}{ Respiratórias } & \multicolumn{2}{c}{ Infecciosas } & \multicolumn{2}{c}{ Externas } & \multicolumn{2}{c}{ Todas } \\
Zonas* & Taxas & RR & Taxas & RR & Taxas & RR & Taxas & RR & Taxas & RR \\
\hline 1 & 22,46 & 1,21 & 8,08 & 1,20 & 2,86 & 2,10 & 10,78 & 1,86 & 67,70 & 1,18 \\
2 & 22,16 & 1,19 & 7,56 & 1,12 & 2,14 & 1,57 & 7,59 & 1,31 & 64,25 & 1,12 \\
3 & 18,78 & 1,01 & 6,97 & 1,03 & 1,92 & 1,41 & 6,76 & 1,17 & 58,02 & 1,01 \\
4 & 18,63 & 1,00 & 6,74 & 1,00 & 1,36 & 1,00 & 5,79 & 1,00 & 57,24 & 1,00 \\
\hline
\end{tabular}

Fontes: Fundação SEADE ${ }^{13}$ (1992), STEPHENS, e col. ${ }^{23}$ (1994).

* Confira Tabela 1

RR - Risco relativo

Tabela 9 - Sobremortalidade para a faixa etária 0-4 anos por zonas.

\begin{tabular}{|c|c|c|c|c|}
\hline Mortalidade & Zona* 1 & Zona* 2 & Zona*3 & Zona* 4 \\
\hline Mortes observadas (a) & 3.143 & 1.484 & 523 & 265 \\
\hline Mortes esperadas (b) & 1.409 & 944 & 355 & 265 \\
\hline Sobremortalidade relativa (c) & 1.734 & 540 & 168 & 0 \\
\hline (c) / (a) & $55 \%$ & $36 \%$ & $32 \%$ & 0 \\
\hline
\end{tabular}

* Confira Tabela 1 
Tabela 10. - Sobremortalidade das doenças infecciosas e parasitárias para a faixa etária 0-4 anos por zonas.

\begin{tabular}{lcccc}
\hline Mortalidade & Zona* 1 & Zona* & Zona*3 & Zona* 4 \\
\hline Mortes observadas (a) & 326 & 110 & 56 & 13 \\
Mortes esperadas (b) & 74 & 50 & 19 & 14 \\
Sobremortalidade relativa (c) & 252 & 60 & 37 & 0 \\
(c) / (a) & $77 \%$ & $55 \%$ & $66 \%$ & 0 \\
\hline
\end{tabular}

* Confira Tabela 1

A Tabela 10 mostra os resultados da sobremortalidade em relação ao grupo das doenças infecciosas e parasitárias para a faixa etária de 0 a 4 anos.

Da mesma forma, $77 \%$ das mortes por doenças infecciosas, em idades de 0 a 4 anos, poderiam ter sido evitadas na Zona 1 se ela tivesse as mesmas condições socioeconômicas e de ambiente da Zona 4. Para a cidade como um todo, 349 mortes poderiam ter sido evitadas, se as Zonas 1, 2 e 3 tivessem as mesmas condições da Zona 4.

A Tabela 11 mostra a sobremortalidade relativa (mortes evitáveis) para vários grupos etários e causas onde a maioria das mortes podem ser consideradas precoces.

A Tabela 11 mostra que na Zona 1 encontram-se os maiores percentuais de mortes evitáveis. Ações de saúde que pudessem evitar as diarréias e as pneumonias teriam impacto significativo na redução da morte de crianças nessa zona, e que a priorização de medidas que pudessem conter os homicídios poderiam resultar em redução importante na morte precoce de adultos jovens. Programas que enfrentassem as causas das doenças cardíacas poderiam também ter impacto importante na morte precoce de adultos entre 45 e 64 anos. A Tabela 11 mostra ainda que medidas preventivas para evitar acidentes de trânsi- to teriam maior efetividade se dirigidas à população de 45-64 anos. O quadro apresentado pode servir de base para um programa de saúde municipal.

\section{Comentários Finais e Recomendações para Políticas de Saúde}

Esse tópico final apresenta os principais achados da pesquisa de campo em São Paulo. A análise da sobremortalidade ("excess mortality") entre as zonas socioeconômicas e de ambiente é sugerida com um instrumento útil para ressaltar áreas, grupos etários e causas de doenças susceptíveis de intervenções dos poderes públicos. Propõe-se alternativas para uso futuro de dados.

São Paulo é conhecida como uma das mais bem sucedidas cidades do mundo em desenvolvimento: tem o mercado financeiro mais dinâmico da América Latina, associado a um distrito industrial eficiente, e um setor de serviços altamente sofisticado. É uma das cinco cidades do mundo onde o helicóptero é mais utilizado para transporte privado. Contudo, sua força econômica não foi capaz de fornecer padrão decente de vida para uma grande parcela de seus habitantes. Do total da população paulistana, 5.664 .000 pessoas ou $59 \%$, vivem em habitações precárias $\left(\mathrm{Jacobi}^{14}, 1990\right)$ e há, ainda, 1.536 .000 (16\%) sem nenhum acesso a

Tabela 11 - Sobremortalidade relativa entre as zonas para várias causas e faixas etárias.

\begin{tabular}{|c|c|c|c|}
\hline Causas de mortalidade e faixas etárias & Zona* 1 & Zona* 2 & Zona*3 \\
\hline $\begin{array}{l}\text { 0-4 anos } \\
\text { (infecciosas) }\end{array}$ & $77 \%$ & $55 \%$ & $66 \%$ \\
\hline $\begin{array}{l}\text { 0-4 anos } \\
\text { (diarréias) }\end{array}$ & $83 \%$ & $58 \%$ & $76 \%$ \\
\hline $\begin{array}{l}0-4 \text { anos } \\
\text { (respiratórias) }\end{array}$ & $74 \%$ & $53 \%$ & $55 \%$ \\
\hline $\begin{array}{l}0-4 \text { anos } \\
\text { (pneumonias) }\end{array}$ & $76 \%$ & $57 \%$ & $60 \%$ \\
\hline $\begin{array}{l}15-44 \text { anos } \\
\text { (externas) }\end{array}$ & $58 \%$ & $28 \%$ & $22 \%$ \\
\hline $\begin{array}{l}15-44 \text { anos } \\
\text { (homicídios) }\end{array}$ & $66 \%$ & $42 \%$ & $35 \%$ \\
\hline $\begin{array}{l}\text { 15-44 anos } \\
\text { (acidente de trânsito) }\end{array}$ & $11 \%$ & $13 \%$ & $7 \%$ \\
\hline $\begin{array}{l}\text { 45-64 anos } \\
\text { (doenças do coração) }\end{array}$ & $42 \%$ & $37 \%$ & $23 \%$ \\
\hline $\begin{array}{l}\text { 45-64 anos } \\
\text { (doenças cérebro-vasculares) }\end{array}$ & $57 \%$ & $44 \%$ & $26 \%$ \\
\hline $\begin{array}{l}45-64 \text { anos } \\
\text { (acidentes de trânsito) }\end{array}$ & $44 \%$ & $32 \%$ & $2 \%$ \\
\hline
\end{tabular}

* Confira Tabela 1 
redes de esgoto*. Numa cidade que necessita de classe operária e trabalhadores qualificados para fornecer, tanto à indústria quanto aos serviços, padrões crescentes de qualidade, observa-se que 3.168 .000 de pessoas ou $33 \%$ de seus cidadãos são ainda analfabetos e/ou com instrução primária incompleta. Embora a maioria dos domicílios tenha água encanada, há grande variação no consumo per capita entre as zonas.

O zoneamento mostrou que $43,8 \%$ da população da cidade de São Paulo vive em áreas com a pior renda per capita. Os dados de saúde demonstraram que pessoas vivendo nessas áreas tinham padrão de mortalidade mais elevado que aquelas vivendo em áreas com melhores condições socioeconômicas.

Mortes por doenças infecciosas e respiratórias formam importante grupo em idades inferiores a cinco anos. Os diferenciais entre zonas são surpreendentes. Taxas de mortalidade por doenças infecciosas e respiratórias na Zona 1 são maiores do que na Zona 4, respectivamente 3,8 e 4,4 vezes. Diarréia representa $48 \%$ das mortes por doenças infecciosas e a pneumonia forma a maior parte de causas respiratórias, com $87 \%$ de todas as mortes. Apesar de representarem somente $2,6 \%$ de todas as mortes em 1992, esses números provam que a cidade ainda não foi capaz de eliminar as mortes evitáveis através de medidas muito simples.

No grupo etário 15-44 anos há claros sinais de que a cidade transformou-se em cenário de uma "epidemia" de violência. Em 1992 foram enumeradas 3.750 mortes por homicídios, a taxa chegando a 4 por 10.000 . Se forem considerados somente homens no grupo de 15-24 anos, sobe-se para 19,5 por 10.000 , bem abaixo dos Estados Unidos, com 2,19 por 10.000, a mais alta taxa masculina nesse grupo etário entre outras nações industrializadas (Cohen e Swift ${ }^{5}$, 1993). Esses números evidenciam problema de difícil solução, por causa das ferramentas tradicionais disponíveis no setor da saúde. Acredita-se que somente ações conjun- tas entre vários órgãos municipais, estaduais e nacionais poderiam enfrentar essa questão.

O grupo etário 45-64 anos apresenta fatos interessantes. É de praxe afirmar que altas taxas de doenças do sistema circulatório indicam transição das doenças da pobreza para as da riqueza. Os dados de São Paulo mostram padrão diferente, onde as Zonas 1 e 2, de piores condições socioeconômicas e de ambiente, apresentam taxas mais altas que as das Zonas 3 e 4 . Acredita-se que essa descoberta deve ser aprofundada em futuras pesquisas.

Finalmente, são apresentadas algumas sugestões para uso futuro dos dados. Sugere-se que as variáveis utilizadas para compor o índice podem estar representando medidas conservadoras de carência, já que foram escolhidas sob influência do indicador mortalidade e suas tradicionais associações em vez de escolher variáveis que demonstrassem padrões mais nítidos de desigualdade e exclusão social**.

A habitação precária é um dos principais problemas das pessoas que vivem em São Paulo, como já foi assinalado, e os cortiços são um dos exemplos dessa realidade. Uma pesquisa domiciliar, realizada em 1985, visitou 65 cortiços e detectou semlhanças em relação à sua precariedade, mas também complexidades e grande variedade de diferenças (Sempla ${ }^{21}$, 1985). Essa pesquisa sugere que quaisquer conclusões ou inferências sobre o assunto são precipitadas, pois a realidade dos cortiços é pouco conhecida. Novos indicadores deveriam ser explorados a fim de caracterizar melhor esse tipo de habitação. Os indicadores tradicionais tais como utilização de água, rede de esgotos e número de pessoas por casa não puderam apontar com clareza áreas com maior concentração de cortiços. Associado a isso, é imperativo que se inicie um diálogo entre os vários bancos de dados de diferentes instituições. Esses são, com frequiência, baseados em diferentes áreas geográficas e sempre possuem objetivos distintos.

\footnotetext{
*Dados dos arquivos internos da SABESP, 1993.

*** Variáveis tais como, "nível de desemprego" ou "metro quadrado construído per capita" poderiam cumprir esse papel. Entretanto, pode-se contestar também a representatividade empírica dessas variáveis pois partem de dados sobre a cidade "legal" e não sobre a "real". Isso se dá porque a maioria dos bancos de dados oficiais não computam dados de mercado informal e construções clandestinas.
} 


\section{REFERÊNCIAS BIBLIOGRÁFICAS}

1. AKERMAN, M.; STEPHENS, C.; CAMPANÁRIO, P.; MAIA, P.B. Saúde e meio ambiente: uma análise de diferenciais intra-urbanos enfocando o Município de São Paulo, Brasil. Rev. Saúde Pública, 28: 320-5, 1994.

2. BRADLEY, D.; STEPHENS, C.; HARPHAM, T.; CAIRNCROSS, S. A review of environmental health impacts in developing countries cities. Washington, World Bank Urban Management Program, 1992.

3. BUSSAB, W.O. \& DINI, N.P. Pesquisa de emprego e desemprego. SEADE/DIEESE: regiões homogêneas da Grande São Paulo. S. Paulo Perspec., 1:5-11, 1985.

4. CARSTAIRS, V. \& MORRIS, R. Deprivation and mortality: an alternative to social class? Community Med., 11: 210-9, 1989.

5. COHEN, L. \& SWIFT, S. A public health approach to the violence epidemic in the United States. Environ. Urban., 5: 50-66, 1993.

6. COMUNE, A.E. et al. Indicadores de qualidade de vida. In: Longo, C.A. \& Rizzieri, J.A.B. org. Economia urbana: custos de urbanização e finanças públicas. São Paulo, IPE/ USP, 1982.

7. FERREIRA, C.E.C. \& GRILLO, J.E. Níveis e padrões da mortalidade infantil nas sub-regiões do Estado de São Paulo. Inf. Demogr., 8: 137-54, 1983.

8. FERRERA, C.E.C. \& SAAD, P. A questão da mortalidade. In: Fundação SEADE. O jovem na Grande São Paulo. São Paulo, 1988. p. 119-49.

9. FERREIRA, C.E.C. Mortalidade infantil: a manifestação mais cruel das desigualdades sociais. S. Paulo Perspec., 3 24-9, 1989.

10. FUNDAP - FUNDAÇÃO DE DESENVOLVIMENTO ADMINISTRATIVO. Características regionais de saúde no Estado de São Paulo. São Paulo, 1983.

11. FUNDAÇÃO SEADE. O jovem na Grande São Paulo. São Paulo, 1988.

12. FUNDAÇÃO SEADE. O idoso na Grande São Paulo. São Paulo, 1990.

13. FUNDAÇÃO SEADE. Pesquisa de condições de vida na Região Metropolitana de São Paulo: renda, educação, habitação e principais resultados. São Paulo, 1992.
14. JACOBI, P. Habitat and health in the municipality of São Paulo. Environ. Urban., 2: 33-45, 1990.

15. LESER, W.S.P. Relacionamento de certas características populacionais com a mortalidade infantil no município de São Paulo. Probl. Bras., 10: 17-33, 1972.

16. LOCKER, D. Mensuring social inequality in dental health services research: individual, household and area based measures. Toronto, Department of Comunity Dentistry and Community Dental Health Services Research Unit, Faculty of Dentistry, University of Toronto, 1992.

17. MONTEIRO, C.A. Contribuição para o estudo do significado da evolução do coeficiente da mortalidade infantil no Município de São Paulo (SP), Brasil nas três últimas décadas (1950-79). Rev. Saúde Pública, 16: 7-18, 1982.

18. MONTEIRO, C.A. \& BENICIO, M.H.A. Estado nutricional e renda. S. Paulo Perspec., 1: 67-70, 1987.

19. RAMOS, L.R. \& GOIHMAN, S. Geographical stratification by socio-economic status: metodology from a household survey with elderly people in S. Paulo, Brazil. Rev. Saúde Pública, 23: 478-92, 1989.

20 SECRETARIA DE PLANEJAMENTO E GESTÃO. Como estão os paulistas? Indicadores de renda per capita e condições sociais. In: Secretaria de Planejamento e Gestão. Como está São Paulo? São Paulo, 1993.

21. SECRETARIA DE PLANEJAMENTO DO MUNICÍPIO DE SÃO PAULO. Cortiços em São Paulo: frente e verso. São Paulo, (SEMPLA), 1985.

22. SOBRAL, H.R. Mapeamento das causas de morte no Município de São Paulo: subsídios a uma geografia médica da cidade. Bol. Paul. Geogr., 66: 85-96, 1988.

23. STEPHENS, C.; TIMAEUS, I.; AKERMAN, M.; AVIE, S.; MAIA, P.B.; CAMPANÁRIO, P.; DOE, B.; LUSH, L.; TETTEH, D. Environment and health in developing countries: an analysis of intra-urban mortality differentials using existing data in Accra (Ghana) and Sao Paulo (Brazil) and analysis of urban data of four demographic and health surveys. London, London School of Hygiene and Tropical Medicine, 1994.

24 UNITED NATIONS. Human development report. New York, Oxford University Press, 1992. 